\title{
Notas de Política Educativa
}

2020-05-03

\section{Notas de Política Educativa}

\author{
Centro de Liderazgo y Excelencia Docente (CLED)
}

Follow this and additional works at: https://ciencia.lasalle.edu.co/npe

\section{Citación recomendada}

(CLED), Centro de Liderazgo y Excelencia Docente (2020) "Notas de Política Educativa," Notas de Política Educativa: No. 1 , Article 2.

Disponible en: DOI: https://doi.org/10.19052/npe.2021.1

This Artículo de Divulgación is brought to you for free and open access by the Revistas de divulgación at Ciencia Unisalle. It has been accepted for inclusion in Notas de Política Educativa by an authorized editor of Ciencia Unisalle. For more information, please contact ciencia@lasalle.edu.co. 


\section{Notas de política educativa}

\begin{abstract}
Abril de 2021
Principales resultados

Mejorar la educación rural es una urgencia y un anhelo nacional al que muchos actores aportan sin lograr sinergias efectivas.

Aunque el país cuenta con una planta docente y un número de sedes suficiente, la infraestructura y el fortalecimiento de las capacidades humanas de los directivos $y$ docentes son asuntos urgentes por atender.

Existe una multitud de experiencias educativas creadas para mejorar la educación rural, pero no han sido sistematizadas suficientemente para poder ser replicadas y escaladas.

El país necesita de una política de educación que atienda las particularidades de los territorios rurales. Esta política debe ser integral, intersectorial y ampliamente participativa.
\end{abstract}

Acerca de los investigadores principales:

\section{Wilson Acosta Valdeleón}

Doctor en educación. Director del Centro de Liderazgo y Excelencia Docente

Cristhian James Díaz Meza

Doctor en educación y liderazgo. Vicerrector académico

Jaime Alberto Rendón Acevedo

Doctor en economía internacional y desarrollo.

Centro de Estudios e Investigaciones Rurales (CEIR)

\section{Guillermo Londoño Orozco}

Doctor en educación y sociedad. Decano de la Facultad de Ciencias de la Educación

Myriam Alba Zapata Jiménez

Doctora en estudios latinoamericanos. Directora del

Grupo Educación y Sociedad

Mirta Yolima Gutiérrez Ríos

Doctora Docente investigadora del Doctorado en

Educación y Sociedad

\section{Acerca del estudio}

El estudio es el resultado del convenio de apoyo no. 1107849 de 2019 entre el Ministerio de Educación Nacional y La Universidad de La Salle.

\section{Lineamientos estratégicos para una política integral de educación rural}

Esta nota reporta los principales resultados del proceso de construcción participativa de los lineamientos estratégicos para una política integral de educación rural realizada mediante convenio entre el MEN y la Universidad de La Salle en 2019. El estudio se concentró en comprender los aportes de entidades públicas y privadas a este tema, realizar un mapeo dinámico de la educación rural en el momento del estudio, identificar los factores clave de las experiencias de referencia en educación rural, y establecer los aportes de los actores de la educación rural para su mejoramiento. Como fruto de este estudio, se proponen lineamientos para la construcción de una política integral de educación rural para el país.

\section{Antecedentes}

La educación de los niños, adolescentes y jóvenes en los ámbitos rurales ha sido una preocupación del país expresada en una multiplicidad de acciones desarrolladas por el Estado, la academia y la sociedad civil. La apertura económica de los años 90 condujo a un deterioro del sector rural agropecuario. En respuesta a esta situación, se llegó en el año de 1996 al Pacto Social Rural, en medio del cual se impulsó el diseño del Proyecto de Educación Rural (PER 1) en su primera fase (2000 y 2006), que se centró principalmente en la ampliación de la cobertura, sobre todo en los niveles de la educación básica, y en fortalecer e implementar los modelos pedagógicos flexibles.

La segunda fase (PER 2), que se desarrolló entre 2009 y 2015, se orientó, principalmente, a mejorar los resultados en materia de cobertura, y comenzó a hacer énfasis en la calidad de la educación. Durante esta fase también se priorizó la puesta en marcha de estrategias que contribuyeran, por un lado, a la modernización y consolidación del sector educativo a nivel territorial $y$, por otro, al incremento de la capacidad de gestión de las instituciones que integran los sistemas educativos. 
La terminación del PER II en el año 2015 coincide con los Acuerdos de paz con las FARC. El Punto 1 del documento está relacionado con la educación, en donde se estipulan aspectos concretos como los siguientes: acceso y permanencia en el sistema educativo desde la primera infancia hasta la educación superior; infraestructura educativa; modelos flexibles; gratuidad; formación técnica agropecuaria; eliminación del analfabetismo; becas para la educación superior; formación profesional para la mujer en disciplinas no tradicionales para ella; promoción de la investigación, la innovación y el desarrollo científico y tecnológico para el sector agropecuario en áreas como la agroecología, la biotecnología y los suelos; ampliación de cupos de educación técnica, tecnológica y superior en zonas rurales; y educación superior en áreas relacionadas con el desarrollo rural.

El Estado colombiano también explicita la preocupación por la educación rural en el Plan Decenal de Educación que debe ejecutarse en el periodo 2016-2026. La creación de la Misión para la Transformación del Campo en el gobierno de Juan Manuel Santos, en cabeza del Departamento Nacional de Planeación, propuso un conjunto de políticas de Estado para promover un desarrollo integral -económico, social y ambiental- en el campo colombiano, construido desde el territorio y con una mirada del área rural que va más allá de lo agropecuario. Igualmente, en el Plan Nacional de Desarrollo 2018-2022 Pacto por Colombia, Pacto por la Equidad, se planteó la necesidad de definir una política específica, con el fin de fomentar el desarrollo regional, reducir brechas y mejorar el acceso y la calidad de la educación inicial, preescolar, básica y media en las zonas más apartadas del país.

No ha sido solo el Estado colombiano el que se ha sumado a la construcción de una mejor educación en los territorios rurales. La academia colombiana ha venido participando de larga data en este proceso, y a ella pueden atribuirse los procesos de innovación que culminaron con los modelos flexibles de educación rural. Asimismo, resultan invaluables los procesos de producción de conocimiento que se han producido sobre el desarrollo rural en el país, en medio de los cuales la educación juega un papel altamente relevante. En lo que refiere a la educación superior, es de destacar el esfuerzo realizado por universidades públicas y privadas, y por el SENA, para formar profesionales y técnicos demandados en labores agropecuarias. Al respecto, el proyecto Utopía de la Universidad de La Salle merece una nota especial, puesto que es referente en educación rural superior en el país.
Las organizaciones de la sociedad civil también han aportado a la construcción de una mejor educación rural. El Programa de Desarrollo y Paz del Magdalena Medio inició su trabajo en octubre de 1995 en cuatro departamentos y 32 municipios. En medio de este proceso, se construyó una concepción integral del territorio desde la cual se desarrolló un trabajo de acción integral en varios frentes, siendo uno de ellos la educación, que aporta elementos para la formación de maestros rurales, y el mejoramiento de sus prácticas, específicamente las que tienen que ver con la construcción de paz y convivencia. Son también destacables las contribuciones que han realizado las organizaciones sociales agrupadas en la Mesa de Educaciones Rurales, que, independientes del accionar institucional estatal, han venido generando en el país encuentros de distintos tipos, liderados por actores de la sociedad civil, para visibilizar los problemas de la educación rural. A ello se suma la intención de plantear la urgencia de contar con una política pública propia con programas y proyectos de impacto, para superar la inequidad que se vive en el sector y, en especial, en su educación.

Las fundaciones empresariales que trabajan en todo el país por la educación también han hecho numerosos aportes al mejoramiento de la educación rural. Solo a modo de ejemplo planteamos dos aportes: el Estudio Sobre Docencia Rural en Colombia: Educar para la Paz en Medio del Conflicto Armado, realizado por la Fundación Compartir en el 2019, y el programa Rectores Líderes Transformadores (RLT) de la Fundación Empresarios por la Educación; programa que ha venido formando, durante una década y desde la idea de liderazgo pedagógico a los directivos de zonas urbanas y rurales, y que hoy por hoy ostenta de una red nacional en la que no solo se intercambian recursos de formación, sino experiencias de liderazgo y gestión.

\section{Situación de la educación rural}

El Departamento Nacional de Planeación (DNP) y la Misión para la Transformación del Campo propusieron una nueva clasificación de la ruralidad por categorías, basándose en la cantidad de población y la densidad. De esta manera, los municipios rurales o rurales dispersos representan el $85 \%$ por área geográfica del territorio, y el $61 \%$ de la cantidad de municipios a nivel nacional, evidenciando que así es la mayor parte del territorio y la mayor cantidad de municipios. Por consiguiente, se podría afirmar que Colombia es un país con mayoría de territorios rurales. 
Mapa1: Colombia con municipios discriminados por categorías de ruralidad

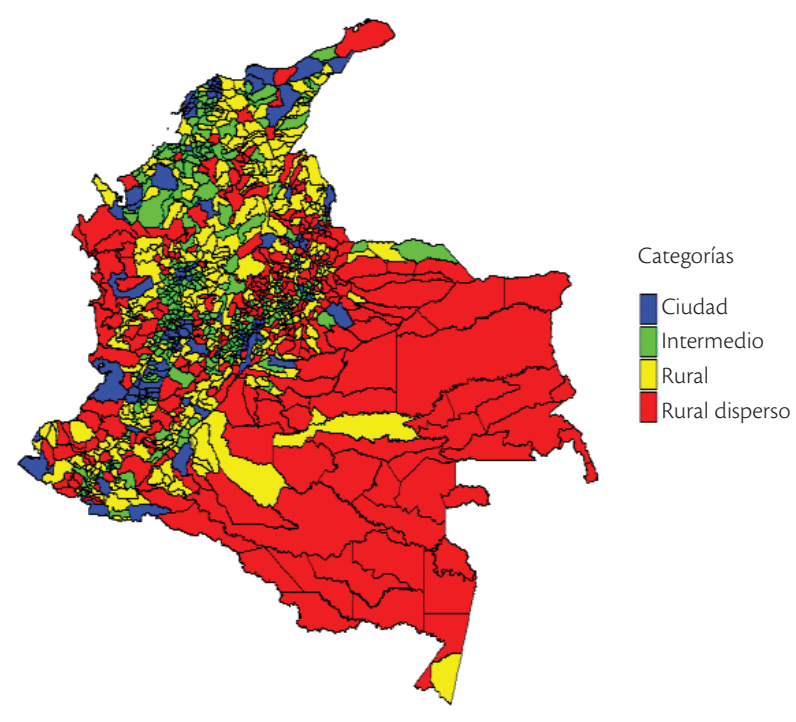

Fuente: elaboración propia con base en la Misión para la Transformación del Campo (2014) y el DANE (2018)

Tabla 1. Cantidad de estudiantes por profesor en zonas urbana y rural 2018

\begin{tabular}{|l|c|c|c|}
\hline Nivel educativo & $\begin{array}{c}\text { Razón: cantidad de estudiantes } \\
\text { por profesor a nivel nacional }\end{array}$ & $\begin{array}{c}\text { Razón: cantidad de estudiantes } \\
\text { por profesor en zona urbana }\end{array}$ & $\begin{array}{c}\text { Razón: cantidad de estudiantes } \\
\text { por profesor en zona rural }\end{array}$ \\
\hline Preescolar & 50 & 51 & 48 \\
\hline Primaria & 30 & 41 & 19 \\
\hline Secundaria y media & 34 & 37 & 26 \\
\hline Total & 31 & 36 & 21 \\
\hline
\end{tabular}

Fuente: elaboración propia con base en SIMAT

Según el SIMAT, para el 2018 los estudiantes matriculados en los niveles formativos de básica primaria, secundaria y media ascendían a 10.104.697, de los cuales el 76 \% (7.692.186) corresponde a estudiantes matriculados en sedes educativas ubicadas en zonas urbanas. En tanto, el $24 \%$ (2.412.511) estudia en zonas rurales. Es decir, de cada cuatro estudiantes en Colombia, uno se encuentra en una zona rural.

Según el SIMAT, los establecimientos ascienden a 19.652 y se distribuyen en todo el territorio nacional en los sectores no oficial y oficial. A nivel nacional, se evidencia que existe el $50 \%$ en cada uno de ambos sectores. Las sedes educativas ascienden a 53.849. Se identifica que para todos los niveles educativos y para los dos sectores existen muchos más estudiantes por sede en la zona ur- bana (428) que en la rural (67). Sin embargo, en el sector oficial la diferencia corresponde a una proporción que es 10 veces mayor; en efecto, la proporción de estudiantes en la zona urbana es de 650 por sede y en la zona rural, de 65 estudiantes por sede. Al parecer, con cifras generales, la infraestructura educativa en la zona rural en términos de la cantidad de sedes educativas es suficiente para atender a todos los estudiantes en los niveles formativos de primaria, secundaria y media. Sin embargo, no sucede lo mismo con las condiciones de infraestructura y la dotación de recursos.

Con relación a la oferta educativa, como se aprecia en la tabla 1, se han calculado unas proporciones relacionadas con la cantidad de estudiantes por profesor. Se puede evidenciar que esta proporción en la zona rural 
es inferior a la de la zona urbana. En efecto, existen en promedio veintiún estudiantes por profesor en la zona rural y 36 en la zona urbana. Esto significa que existe una buena cantidad de docentes en la zona rural para atender una mayor demanda en los niveles de básica primaria, secundaria y media.

Los hallazgos han permitido establecer que la prestación del servicio educativo en la zona rural se ofrece en unas condiciones óptimas en términos de la cantidad de profesores y sedes educativas. Sin embargo, es necesaria una redistribución al interior de la administración de cada sede educativa para ofrecer educación para la primera infancia. Esto obedece a que, como se observa en la tabla 3, la participación de estudiantes en prejardín y jardín es apenas del $0,4 \%$ en la zona rural.
No obstante, pese a que las sedes y profesores son suficientes para atender las necesidades del servicio educativo en las zonas rurales, otros factores inciden en el proceso educativo. Es de anotar que el estado de mantenimiento de las sedes en los territorios rurales es muy bajo, y que las condiciones en que los niños y jóvenes reciben enseñanza se deterioran en la medida en que los estudiantes se alejan de las ciudades y las cabeceras municipales.

Con relación a las tasas de cobertura, se ha identificado que los municipios considerados como rural disperso tienen unas tasas inferiores a las ciudades, como se aprecia en la tabla 4. En efecto, las tasas en estos municipios son inferiores hasta en un $24 \%$ para el caso del nivel medio (urbano: $91 \%$ y rural: $67 \%$ ).

Tabla 2. Cantidad de estudiantes por sede educativa según zonas y sector en 2018.

\begin{tabular}{|l|c|c|c|}
\hline Sector & Total & Zona urbana & Zona rural \\
\hline No oficial & 224 & 224 & 221 \\
\hline Oficial & 180 & 650 & 65 \\
\hline Total & 188 & 428 & 67 \\
\hline
\end{tabular}

Fuente: elaboración propia con base en SIMAT

Tabla 3. Tasas de cobertura brutas por nivel formativo en el 2018

\begin{tabular}{|l|c|c|c|c|c|}
\hline Nivel educativo & Nacional & Ciudades y aglomeraciones & Intermedios & Rural & Rural disperso \\
\hline Preescolar & $82 \%$ & $93 \%$ & $82 \%$ & $85 \%$ & $76 \%$ \\
\hline Primaria & $100 \%$ & $107 \%$ & $96 \%$ & $102 \%$ & $98 \%$ \\
\hline Secundaria & $102 \%$ & $113 \%$ & $103 \%$ & $104 \%$ & $93 \%$ \\
\hline Media & $77 \%$ & $91 \%$ & $79 \%$ & $78 \%$ & $67 \%$ \\
\hline
\end{tabular}

Fuente: elaboración propia con base en SIMAT y DANE

Las diferencias en la calidad de la educación que reciben los niños y jóvenes de establecimientos educativos oficiales urbanos y rurales es significativa. No obstante, ambas presentan un gran desnivel frente a la que reciben los niños de instituciones privadas. Esto muestra que existe una doble brecha en la calidad educativa que debe ser atendida con estrategias integrales que articulen procesos de formación de docentes en los niveles inicial, novel y en ejercicio. Aquello corresponde a reformas cu- rriculares implementadas para actualizar competencias y contenidos, que otorgan así el mejoramiento de los ambientes de aprendizaje, al igual que recursos ideados para el aprendizaje mediado por tecnologías de la información, la comunicación y el aprendizaje.

En lo que refiere al financiamiento de la educación rural, los principales recursos para el sector educativo en las distintas entidades territoriales corresponden a la participación para educación del Sistema General de Parti- 


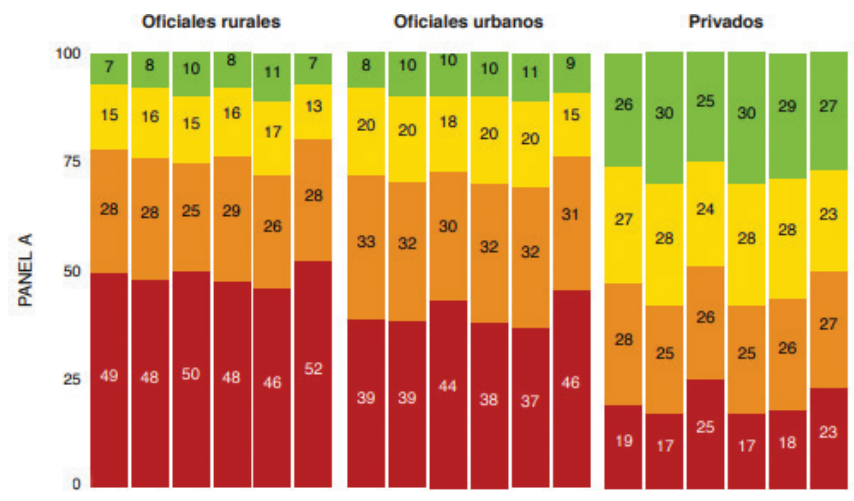

Fuente: Icfes, 2018

Tabla 4. Componentes del gasto en educación del PGN en 2018

\begin{tabular}{|l|c|c|}
\hline Destino del gasto & En billones de pesos & \% de gasto \\
\hline SGP & $\$ 21,7$ & $58 \%$ \\
\hline Fondo Nacional de Prestaciones Sociales del Magisterio & $\$ 8,5$ & $23 \%$ \\
\hline Universidades públicas & $\$ 3,7$ & $10 \%$ \\
\hline Créditos educativos & $\$ 1,5$ & $4 \%$ \\
\hline Alimentación escolar & $\$ 0,85$ & $2 \%$ \\
\hline Otros gastos & $\$ 0,73$ & $2 \%$ \\
\hline Infraestructura & $\$ 0,51$ & $1 \%$ \\
\hline Total & $\$ 37,5$ & $100 \%$ \\
\hline
\end{tabular}

Fuente: elaboración propia a partir de PGN (2018)

cipaciones (SGP). Este incluye recursos de prestación de servicio girados directamente a las entidades territoriales certificadas en educación; cancelaciones giradas a las cuentas de los Fondos de Pensiones Territoriales de los departamentos y del distrito capital, y recursos de Calidad de Matrícula Oficial y de Gratuidad asignados a los distritos, municipios certificados y no certificados en educación (Ministerio de Educación Nacional, 2016).

No existen rubros específicos para la inversión en partidas focalizadas exclusivamente en la educación en la zona rural, de manera que se puedan cerrar las brechas en cobertura y calidad con respecto a la educación en la zona urbana. De esta manera, dejar de invertir en la educación rural consiste ante todo en no incrementar los montos existentes, lo cual podría profundizar las brechas sociales, productivas y de oportunidades que hoy se tie- nen entre el campo y la ciudad. Tales brechas conducen a productividades bajas, pero también a condiciones sociales que ponen en desventaja a la población rural; a un escaso tejido productivo; a la informalidad empresarial y laboral; a incrementar la pobreza, y en especial a dejar al campo, a la Colombia profunda, en unas condiciones en las que solo se les deja a los pobladores la opción del abandono del lugar. Estos se ven obligados a emigrar a las ciudades intermedias, o a tener a las aglomeraciones como única alternativa de vida, en busca de otras oportunidades, aunque aquello sea solo una ilusión. En esencia, la población rural "se la juega" porque nada o muy poco tiene que perder. De tal modo, la educación será uno de esos caminos que el país necesita para resignificar al campo, su producción y sus oportunidades para una vida llevada con dignidad. 


\section{Percepciones de los actores de la educación rural}

La siguiente tabla muestra el resultado de un ejercicio consultivo participativo en el que se realizaron seis mesas territoriales y seis grupos focales con una asistencia de más de quinientas personas, siendo todos ellos actores calificados del sector educativo. En ese escenario, se indagó por nuevas ideas y elementos propios de la educación rural idóneos para aportar a la construcción de los lineamientos de la política pública de educación rural.

\begin{tabular}{|c|c|}
\hline $\begin{array}{l}\text { Equidad, calidad y fortalecimien- } \\
\text { to institucional }\end{array}$ & $\begin{array}{l}\text { Alude a la necesidad de una política de educación rural de alta calidad acorde con las particulari- } \\
\text { dades de cada contexto. Corresponde a una administración adecuada de recursos asignados con } \\
\text { equidad para las regiones. } \\
\text { Apunta al fortalecimiento de la educación rural mediante docentes formados para el sector. Tie- } \\
\text { ne como resultado la creación de contenidos y programas educativos pertinentes e incluyentes } \\
\text { en la educación rural. }\end{array}$ \\
\hline $\begin{array}{l}\text { Trayectorias educativas continuas } \\
\text { y completas }\end{array}$ & $\begin{array}{l}\text { Dan lugar a la ampliación y articulación de la educación básica, media y superior con énfasis en } \\
\text { lo rural. } \\
\text { Permiten la organización de modelos curriculares y evaluativos diferenciados y de pedagogías } \\
\text { rurales flexibles y pertinentes. Se componen de infraestructuras adecuadas y mediaciones que } \\
\text { garantizan la permanencia escolar. Incluyen alianzas estratégicas e incentivos para estudiantes y } \\
\text { docentes rurales. }\end{array}$ \\
\hline $\begin{array}{l}\text { Pedagogías, prácticas y experien- } \\
\text { cias de educación rural }\end{array}$ & $\begin{array}{l}\text { Corresponden a una educación rural pertinente acorde con la diversidad, los sentidos, las realida- } \\
\text { des, las necesidades, los sujetos y los espacios. Consisten en prácticas educativas contextuales y } \\
\text { pertinentes que promueven el intercambio interterritorial. Otorgan reconocimiento, socialización } \\
\text { y fortalecimiento de experiencias educativas innovadoras. Atañen a perspectivas sistémicas que } \\
\text { articulan alianzas público - privadas, así como a la participación comunitaria en planes locales. } \\
\text { Incluyen la flexibilidad como característica esencial de los modelos formativos y de las formas de } \\
\text { organización. }\end{array}$ \\
\hline $\begin{array}{l}\text { Aprendizajes continuos, situados } \\
\text { y diferenciados }\end{array}$ & $\begin{array}{l}\text { Apuntan a una diversidad y autonomía que garantizan el desarrollo de los propios procesos edu- } \\
\text { cativos de las comunidades. Involucran a actores locales como agentes educativos que cumplen } \\
\text { funciones educativas en el contexto comunitario. Incluyen infraestructura y condiciones idóneas } \\
\text { para el derecho a la educación que vinculan a las comunidades. Suponen presupuestos diferencia- } \\
\text { dos que dan prioridad al tema de la equidad por encima del de la eficiencia. }\end{array}$ \\
\hline $\begin{array}{l}\text { Formación de directivos, docentes } \\
\text { y otros agentes }\end{array}$ & $\begin{array}{l}\text { Involucra la formación de docentes y directivos docentes pertinente a los territorios rurales. } \\
\text { Supone la elección, retención y acompañamiento de docentes y directivos docentes que tengan } \\
\text { en cuenta en los perfiles la cercanía al territorio, las necesidades de las escuelas, las situaciones } \\
\text { especiales, y los incentivos que eviten su alta rotación. Incluye las condiciones para el ejercicio } \\
\text { docente que permiten la innovación e investigación en los ámbitos rurales. }\end{array}$ \\
\hline $\begin{array}{l}\text { Prácticas y modelos de liderazgo } \\
\text { y gestión educativa rural }\end{array}$ & $\begin{array}{l}\text { Aluden a la formación de directivos docentes para un liderazgo pedagógico de carácter construc- } \\
\text { tivo, social y comunitario. Suponen modelos de gestión educativa y administrativa que sean arti- } \\
\text { culadores, facilitadores y eficientes. Involucran incentivos al desarrollo de liderazgos innovadores, } \\
\text { contextuales y transformadores. }\end{array}$ \\
\hline
\end{tabular}




\section{Factores claves de las experiencias de referencia en educación rural}

Un análisis colaborativo realizado con diez experiencias educativas que son referentes para la educación rural en Colombia concluyó factores de pertinencia, escalabilidad y replicabilidad a tener en cuenta para la generación de propuestas de educación rural.

\section{Pertinencia}

El reconocimiento social, comunitario e institucional como factor preponderante que surge de la detección de problemáticas coyunturales y de necesidades prioritarias que requieren alternativas de solución.

El reconocimiento social, comunitario e institucional de las condiciones socioculturales $e$ identitarias del territorio, el cual se representa en la recuperación, conservación o resignificación de tradiciones, saberes y prácticas propios del contexto local.

El reconocimiento social, comunitario e institucional de la diversidad de los sujetos sociales, campesinos, afro e indígenas. Se evidencia el carácter vivencial del reconocimiento del otro como sujeto, como comunidad o como ideología en un marco cultural diferenciado e incluyente.

\section{Escalabilidad}

La sostenibilidad sociocultural, ambiental y económica de las experiencias de referencia, que se materializa a partir de alianzas estratégicas como un factor fundamental para la permanencia en el tiempo y la obtención de recursos de diversa índole.

El desarrollo de procesos sistemáticos para el mejoramiento institucional que parte de la autoevaluación institucional y se proyecta en planes de acción concretos, que permiten su consolidación como experiencias con un potencial de implementación cada vez mayor, en su región u otras regiones.

La existencia de horizontes de sentido anclados principalmente a fundamentos axiológicos que posibilitan la legitimidad y el logro de resultados, e impactan, con amplios niveles de reconocimiento por parte de actores sociales, gubernamentales y no gubernamentales, como una oportunidad para su proyección a otros escenarios con características similares.
Replicabilidad

Las disposiciones y capacidades humanas que fortalecen el trabajo en equipo y se constituyen en factores para la motivación, el compromiso y el sentido de pertenencia, al integrarse social y afectivamente en torno a un propósito compartido, vivenciando y disfrutando de encuentros, festivales $y$ actividades institucionales y comunitarias.

El permanente reconocimiento de la gestión que realiza cada persona, desde sus particularidades, posibilidades $y$ desde el rol que desempeña, para hacerla copartícipe de los logros obtenidos.

La autogestión de proyectos educativos integradores y coherentes, por cuanto la comunidad educativa tiene claramente definido su modelo pedagógico y, en coherencia con este, plantea una serie de estrategias pedagógicas como principios de innovación. 


\section{Lineamientos estratégicos para una política \\ de integral de educación rural}

A. Atención educativa a la primera infancia para favorecer el desarrollo humano integral en los niños y niñas.

- Ampliación de la cobertura en la educación inicial (niños y niñas menores de 6 años).

- Formación y asignación de maestros con competencias profesionales específicas para la atención educativa inicial.

- Implementación de estrategias de vinculación del saber de las familias a la práctica pedagógica.

- Adecuación de ambientes pedagógicos para la atención educativa integral en los niños y niñas. Entrega de útiles escolares para garantizar la asistencia y permanencia a los niños y niñas de primera infancia.

B. Atención educativa variada y diversa para favorecer las transiciones entre niveles y la articulación con la educación superior en jóvenes en los ámbitos rurales.

- Definición e implementación de programas de seguimiento a la trayectoria escolar de cada niño, niña y joven.

- Diseño y oferta de programas a estudiantes provenientes de los ámbitos rurales para la educación superior.

- Diseño de estrategias para promover, apoyar y sostener el acceso de los jóvenes rurales a la educación superior.

- Desarrollo de estrategias de articulación entre primaria y secundaria; secundaria y media, y media y superior.

- Atención integral en áreas psicosociales a los jóvenes en pro de su permanencia a lo largo de su trayectoria escolar

C. Bienestar y cualificación de directivos y docentes que favorezcan su permanencia en los ámbitos rurales y que generen mejores condiciones para el desarrollo de sus prácticas pedagógicas.

- Inclusión de estrategias en los programas de formación inicial de docentes para la atención educativa en la ruralidad.

- Fortalecimiento de los programas de formación continuada (PFC) en las Escuelas Normales Superiores.

- Promoción de estrategias colaborativas de formación en servicio para maestros y directivos.

- Implementación de estrategias de apoyo dirigidas a los docentes noveles para su apropiación y permanencia en el territorio.

- Generación de incentivos y condiciones para el bienestar y el desarrollo humano de directivos $y$ docentes en el territorio. 
D. Transformación curricular para asegurar la pertinencia de la educación que reciben los niños, adolescentes y jóvenes en los ámbitos rurales.

- Implementación de estrategias para contextualizar los lineamientos curriculares ante las particularidades de los territorios.

- Definición e implementación de estrategias para el diseño y la gestión de currículos pertinentes.

- Implementación de estrategias curriculares que promueven campos del conocimiento y capacidades diversas.

- Definición e implementación de estrategias para asegurar el enfoque de inclusión en las prácticas educativas.

- Implementación de sistemas flexibles de formación, seguimiento y evaluación de los procesos educativos.

E. Innovación en modelos y estrategias pedagógicas que permiten potencializar las capacidades de niños, adolescentes y jóvenes en los ámbitos rurales.

- Formación de los docentes y estudiantes en ciencia, tecnología e innovación a lo largo de la trayectoria educativa.

- Formación, innovación e investigación educativa de los docentes partiendo del contexto rural.

- Definición e implementación de estrategias para la transferencia de innovaciones pedagógicas a las instituciones educativas rurales.

- Implementación de programas para el acompañamiento a docentes en la utilización de nuevas didácticas.

- Desarrollo de programas de producción de conocimiento colectivo sobre los procesos pedagógicos desarrollados en la ruralidad.

F. Infraestructura física y recursos educativos para asegurar una educación digna y de calidad a los niños, adolescentes y jóvenes en los ámbitos rurales.

- Provisión y revisión periódica de servicios básicos para el funcionamiento de las instituciones educativas rurales.

- Acondicionamiento de la infraestructura de las sedes educativas rurales.

- Implementación de la normativa existente para los internados escolares.

- Entrega y reposición del material didáctico para asegurar aprendizajes significativos $y$ autónomos en los niños.

- Prestación a tiempo y continua de los servicios de alimentación y transporte escolar en los territorios rurales

G. Intersectorialidad y visión global del territorio para la atención educativa en los ámbitos rurales.

- Creación de un subsistema sectorial de educación rural.

- Creación de rutas de atención institucional y sectorial.

- Generación de rutas de articulación y coordinación intersectorial estatal y privada. 


\section{Referencias}

Acuerdo Final. (2016). Acuerdo Final para la terminación del conflicto y la construcción de una paz estable y duradera. Bogotá D.C.

Bautista, M. \& González G. (2019). Docencia rural en Colombia: educar para la paz en medio del conflicto armado. Fundación Compartir: Bogotá D.C.

Departamento Nacional de Planeación. [DNP]. (2014). Bases del Plan Nacional de Desarrollo 2014-2018 "Todos por un Nuevo País". Bogotá D.C.

DNP. (2014). El campo colombiano: un camino hacia el bienestar y la paz. Tomo 1. Bogotá D.C.

DNP. (2018). Documento Conpes 3931. Política nacional para la reincorporación social y económica de exintegrantes de las FARC. Bogotá D.C.

DNP. (2018). Documento Conpes 3932. Lineamientos para la articulación del Plan Marco de Implementación del Acuerdo Final con los instrumentos de planeación, programación, seguimiento a políticas públicas de orden nacional y territorial. Bogotá D.C.

DNP. (2019). Bases del Plan Nacional de Desarrollo 20182022. Pacto por Colombia, pacto por la equidad. Departamento Nacional de Planeación: Bogotá D.C.

DNP. (2019). Plan Nacional de Desarrollo 2018-2022 "Pacto por Colombia, pacto por la equidad". Bogotá D.C.

Econometría. (2015). Producto 4. Informe final ajustado. Evaluación de los resultados de la implementación del Programa de Fortalecimiento de la Cobertura con Calidad para el Sector Educativo Rural PER fase II, en su población beneficiaria identificando los efectos (esperados y no esperados) de la intervención sobre sus variables objetivo. Econometría-SEl: Bogotá D.C.

Freire, P. (2009). La educación como práctica de la libertad. Siglo XXI: Madrid.

Gardner, H. (2011). Inteligencias múltiples. La teoría en la práctica. Paidós: Barcelona.

Ministerio de Educación Nacional. [MEN]. (2002). Decreto 3020 del 2002, Bogotá D.C., 10 de diciembre de 2002.

MEN. (2013). Manual para la formulación y ejecución de Planes de Educación Rural. Bogotá D.C.: Ministerio de Educación Nacional.

MEN. (2019). Misión y visión. [25 de noviembre de 2019]. Recuperado de https://www.mineducacion.gov. co/1759/w3-article-89266.html?_noredirect=1
MEN. (2015a). Colombia territorio rural: apuesta por una política educativa para el campo. Ministerio de Educación: Bogotá D.C.

MEN. (2015b). Decreto 1851 del 2015, Bogotá D.C., 16 de septiembre de 2015.

MEN. (2015c). Modalidades de la educación inicial. Bogotá D. C.: MEN. Recuperado de http://bit.ly/1N$\mathrm{mRlzD}$

MEN. (2015d). Portafolio de modelos educativos. Bogotá D. C.: MEN.

MEN. (2015e). Resolución 200 de 2015, por la cual se regula la administración de los aportes establecidos por la Ley 21 de 1982, se definen los criterios para su inversión, se establece el procedimiento para obtener la cofinanciación de proyectos de infraestructura educativa y se deroga la Resolución 7650 de 2011, Bogotá D.C, 5 de enero de 2015. Recuperado de http://bit.ly/1M6GMNS

MEN. (2016). Plan Especial de Educación Rural. (Segundo borrador). Bogotá D. C.: MEN.

MEN. (2017a). Decreto 1421. Reglamenta en el marco de la educación inclusiva la atención educativa a la población en discapacidad, Bogotá D.C., 29 de agosto de 2017.

MEN. (2017b). Plan Decenal de Educación 2016-2026. El camino hacia la calidad y la equidad. Bogotá D.C.

MEN. (2017c). Plan Especial de Educación Rural. Hacia el desarrollo rural y la construcción de paz. (Documento borrador). Versión del 14 de junio de 2017. Bogotá D.C.: Ministerio de Educación Nacional.

MEN. (2017d). Plan Nacional Decenal de Educación 20162026. El camino hacia la calidad y la equidad. Bogotá D.C.: MEN.

MEN. (2018). Decreto 1416, Reglamentación de la Ley 1804 del 2016 sobre política de Estado para el desarrollo integral de la primera infancia, Bogotá D.C., 3 de agosto de 2018.

MEN. (2019). Plan Integral de Educación Rural. (Documento en revisión). Bogotá D.C.: Ministerio de Educación Nacional.

MEN \& Proyecto de Educación Rural. [PER]. (2008). Al tablero. N. . . 45. Recuperado de https://www.mineducacion.gov.co/1621/propertyvalue-38198.html

MEN \& UNICEF. (2015a). Lineamientos de política pública para la atención integral de niños, niñas y adolescentes vinculados al sistema educativo formal en 
la modalidad de internados escolares con enfoque diferencial. Bogotá: MEN \& UNICEF.

MEN \& UNICEF. (2015b). Manual de funcionamiento y administración de los internados escolares. Bogotá: MEN \& UNICEF.

MEN \& Viceministerio de Educación Preescolar, Básica y Media. (2015). Colombia territorio rural. Apuesta por una política educativa para el campo. Bogotá: MEN \& Viceministerio de Educación Preescolar, Básica y Media.

MEN \& Viceministerio de Educación Superior. (2013). Lineamientos de política de educación superior inclusiva. Bogotá: MEN \& Viceministerio de Educación Superior.

MEN \& Viceministerio de Educación Preescolar, Básica y Media, Nacional y Visión Social. (2015). Colombia territorio rural: apuesta por una política educativa para el campo. Bogotá D.C.

Ministerio del Interior. [s.f.]. El enfoque diferencial y étnico en la política pública de víctimas del conflicto armado. Bogotá D.C.: Ministerio del Interior.

Monereo, C. (1999). Estrategias de enseñanza y aprendizaje. Formación del profesorado y aplicación en la escuela. Barcelona: Grao.

Nussbaum. M. (2012). Crear capacidades. Propuestas para el desarrollo humano. Barcelona: Paidós.

Senn, A. (2012). Desarrollo y libertad. XI reimpresión. Bogotá D.C.: Editorial Planeta.

OEI. (2009). Las trayectorias escolares. Del problema individual al desafio de la política educativa. Buenos Aires: Ministerio de Educación de la Nación.

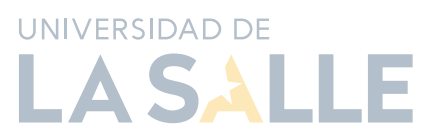

El Centro de Liderazgo y Excelencia Docente

(CLED) tiene como misión generar y difundir conocimiento útil y transferible por medio de procesos de investigación, desarrollo e innovación de alta calidad que buscan fortalecer el liderazgo directivo y la docencia de excelencia en las instituciones educativas desde los ideales de calidad, pertinencia, visibilidad, estabilidad y respeto.

\section{Comité editorial}

Leonor Botero Arboleda

Vicerrectora de Investigación y Transferencia

Cristhian James Díaz Meza FSC

Vicerrector Académico

Wilson Acosta Valdeleón

Director del Centro de Liderazgo y Excelencia Docente

Jaime Alberto Rendón Acevedo

Director del Centro de Estudios e Investigaciones

Rurales (CEIR)

Carlos Valerio Echavarría

Director del Doctorado en Educación y Sociedad

\section{Producción editorial}

Alfredo Morales Roa

Dirección editorial

Rosa González

Coordinación editorial

Ricardo Andrés Manrique

Corrección de estilo

William Yesid Naizaque Ospina

Diseño y diagramación 
